\title{
SOLUTION OF 1D AND 2D POISSON'S EQUATION BY USING WAVELET SCALING FUNCTIONS
}

\author{
R. B. Burgos ${ }^{\mathrm{a}}$, \\ and H. F. C. Peixoto \\ ${ }^{a}$ Universidade do Estado do Rio de Janeiro \\ Departamento de Estruturas e Fundações \\ Rua S. Francisco Xavier, 524, \\ Rio de Janeiro, RJ, Brasil \\ rburgos@eng.uerj.br \\ ${ }^{\mathrm{b}} \mathrm{PUC}-\mathrm{Rio}$ \\ Departamento de Engenharia Civil \\ Rua Marquês de São Vicente, 225 \\ Rio de Janeiro, RJ, Brasil. \\ hfcpeixoto@gmail.com \\ Received: September 27, 2016 \\ Revised: October 14, 2016 \\ Accepted: November 07, 2016
}

ABSTRACT

The use of multiresolution techniques and wavelets has become increasingly popular in the development of numerical schemes for the solution of partial differential equations (PDEs). Therefore, the use of wavelet scaling functions as a basis in computational analysis holds some promise due to their compact support, orthogonality and localization properties. Daubechies and Deslauriers-Dubuc functions have been successfully used as basis functions in several schemes like the WaveletGalerkin Method (WGM) and the Wavelet Finite Element Method (WFEM). Another possible advantage of their use is the fact that the calculation of integrals of inner products of wavelet scaling functions and their derivatives can be made by solving a linear system of equations, thus avoiding the problem of using approximations by some numerical method. These inner products were defined as connection coefficients and they are employed in the calculation of stiffness matrices and load vectors. In this work, some mathematical foundations regarding wavelet scaling functions, their derivatives and connection coefficients are reviewed. A scheme based on the Galerkin Method is proposed for the direct solution of Poisson's equation (potential problems) in a meshless formulation using interpolating wavelet scaling functions (Interpolets). The applicability of the proposed method and some convergence issues are illustrated by means of a few examples.

Keywords: wavelets, Poisson's equation, Wavelet-Galerkin Method

\section{NOMENCLATURE}

\section{A}

$a_{k} \quad k^{\text {th }}$ Daubechies filter coefficient, dimensionless

$c_{k} \quad k^{\text {th }} \quad$ Deslauriers-Dubuc filter coefficient, dimensionless

$d \quad$ interpolation coefficient

d vector of interpolation coefficients in 1D problems

EA axial stiffness, $\mathrm{kN}$

$F \quad$ force, $\mathrm{kN}$

f load vector

FDM Finite Differences Method

g matrix of boundary conditions

I identity matrix

$i \quad$ wavelet translation

$j \quad$ wavelet translation

k stiffness matrix

$k \quad$ wavelet translation, integer value

$M \quad$ wavelet moments

$N \quad$ wavelet order

P matrix of filter coefficients

$R \quad$ reaction force, $\mathrm{kN}$

$u \quad$ displacement, $\mathrm{m}$

WGM Wavelet-Galerkin Method

\section{Greek symbols}

$\Gamma \quad$ vector of wavelet connection coefficients

$\Phi \quad$ vector scaling function evaluations

$\Lambda \quad$ vector of derivative values $\boldsymbol{\alpha}$ vector of interpolation coefficients in 2D problems

$\delta \quad$ Kronecker delta

$\eta \quad$ dimensionless coordinate in $y$ direction

$\varphi \quad$ scaling function

$\lambda \quad$ vector of Lagrange multipliers

$\sigma \quad$ variance

$\xi \quad$ dimensionless coordinate in $x$ direction

$\psi \quad$ wavelet function

\section{Subscripts}

$\begin{array}{ll}i & \text { index of summation } \\ j & \text { index of summation } \\ k & \text { index of summation } \\ p & \text { particular solution }\end{array}$

\section{Superscripts}

$j \quad$ wavelet level of resolution

$m$ polynomial order

$n \quad$ order of derivation

\section{INTRODUCTION}

The use of wavelet-based numerical schemes has become increasingly popular in the last three decades. Wavelet scaling functions have several properties that are especially useful for representing solutions of differential equations (DE's), such as orthogonality, compact support and a certain number 
of vanishing moments (exact representation of polynomials). Their capability of representing data at different levels of resolution allows the efficient and stable calculation of functions with high gradients or singularities and their incorporation in numerical methods.

A complete basis of wavelets can be generated through dilation and translation of a mother scaling function. Although many applications use only the wavelet filter coefficients of the multiresolution analysis, there are some which explicitly require the values of the basis functions and their derivatives, such as the Wavelet Finite Element Method (WFEM) (Ma et al., 2003).

Compactly supported wavelets and scaling functions have a finite number of derivatives which can be highly oscillatory. This makes the numerical evaluation of integrals of their inner products difficult and unstable. Those integrals are called connection coefficients and appear naturally when applying a numerical method for the solution of a DE. Due to some properties of scaling functions, these coefficients can be obtained by solving an eigenvalue problem using filter coefficients.

The most commonly used wavelet family is the one developed by Ingrid Daubechies (1988). All the mathematical foundations were formulated for Daubechies wavelets and then extended to other families. Working with dyadically refined grids, Deslauriers and Dubuc (1989) obtained a new family of functions with interpolating properties, later called Interpolets. Unlike Daubechies, Interpolets are symmetric, which is especially interesting in numerical analysis.

The use of Interpolets instead of Daubechies scaling functions greatly improves the method's accuracy (Burgos et al., 2015). One and two dimensional examples were proposed and the convergence of the method was studied.

\section{WAVELET SCALING FUNCTIONS}

Multiresolution analysis using orthogonal, compactly supported wavelets has been successfully applied in numerical simulation. Wavelet basis are composed of two kinds of functions: scaling functions $(\varphi)$ and wavelet functions $(\psi)$. The two combined form a complete Hilbert space of square integrable functions. The spaces generated by scaling and wavelet functions are complementary and both are based on the same mother function.

In the following expressions, known as the twoscale relation, $a_{k}$ are the scaling function filter coefficients and $N$ is the wavelet order.

$$
\varphi(x)=\sum_{k=0}^{N-1} a_{k} \varphi(2 x-k)=\sum_{k=0}^{N-1} a_{k} \varphi_{k}(2 x)
$$

$$
\psi(x)=\sum_{k=0}^{N-1}(-1)^{k} a_{N-1-k} \varphi(2 x-k)
$$

In general, there are no analytical expressions for wavelet scaling functions, which can be obtained using iterative procedures like Eqs. (1) and (2). In order to comply with the requirements of orthogonality and compact support, they present, in general, an irregular fractal-like shape. An example of this irregular shape is shown in Fig. 1, which presents Daubechies' scaling function of order $N=4$.

\section{Wavelet properties}

The set of properties summarized in the following expressions is valid for Daubechies wavelets but can be adapted to other wavelet families, such as Delauriers-Dubuc Interpolets. Some of these properties, like compact support and unit integral, are required for the use of the wavelet family in numerical methods. Others, like orthogonality, are desirable but not extremely necessary.

$$
\begin{aligned}
& \operatorname{supp}(\varphi)=\left[\begin{array}{ll}
0 & N-1
\end{array}\right], \\
& \int_{-\infty}^{+\infty} \varphi(x) d x=1, \\
& \int_{-\infty}^{+\infty} \varphi(x-i) \varphi(x-j) d x=\delta_{i, j}, \\
& x^{m}=\sum_{-\infty}^{+\infty} c_{k}^{m} \varphi(x-k), \quad m \leq N / 2-1 .
\end{aligned}
$$

The last expression in Eq. (3) derives from the vanishing moments property, which states that a set of shifted and scaled Daubechies wavelets of order $N$ is capable of representing exactly an $N / 2-1$ degree polynomial.

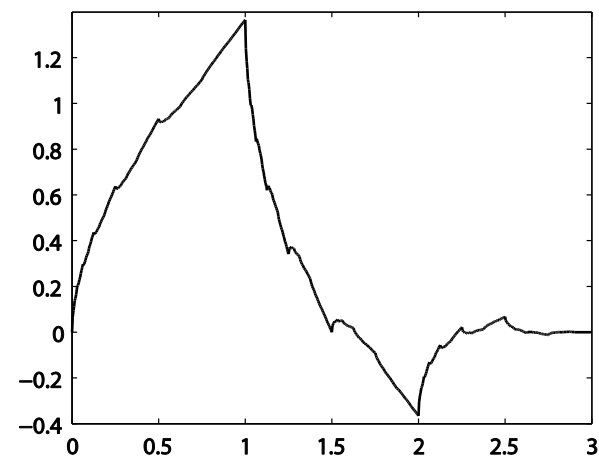

Figure 1. Daubechies scaling function of order $N=4$.

\section{Wavelet derivatives}

In the process of solving a DE using numerical methods, derivatives of the basis functions tend to appear. As there are no analytical expressions for scaling functions, derivatives are obtained in dyadic 
grid points and the refinement of the solution depends on the level of resolution needed (Lin et al., 2005). The scale relation can be differentiated $n$ times, generating the following expression:

$$
\varphi^{(n)}(x)=2^{d} \sum_{i=0}^{N-1} a_{i} \varphi^{(n)}(2 x-i)
$$

Applying Eq. (4) to integer points results in the following system of equations shown in matrix form.

$$
\left(2^{n} \mathbf{A}-\mathbf{I}\right) \boldsymbol{\Lambda}^{(n)}=0, \quad \mathbf{A}=\left[a_{2 i-k}\right]_{0 \leq i, k \leq N-1}
$$

In Eq. (5), $\mathbf{A}$ represents the filter coefficients matrix, $\mathbf{I}$ is the identity matrix and $\Lambda^{(n)}$ is the vector containing derivative values at integer points of the grid. Equation (5) is an eigenvalue problem which, for unique solution, has to be normalized using the so-called moment equation, derived from the property of exact polynomial representation. This equation is given by Latto et al. (1992) and provides a relation between derivative values at integer points.

$$
\begin{aligned}
& n !=\sum_{i=0}^{N-1} M_{i}^{n} \varphi^{(n)}(x-i) \\
& M_{i}^{n}=\frac{1}{2^{j+1}-2} \sum_{k=0}^{n}\left(\begin{array}{l}
n \\
k
\end{array}\right) i^{n-k} \sum_{l=0}^{k-1}\left(\begin{array}{l}
k \\
l
\end{array}\right) M_{0}^{l}\left(\sum_{i=0}^{N-1} a_{i} i^{k-l}\right)
\end{aligned}
$$

In Eq. (6), the coefficient $M$ is the moment of the $n^{\text {th }}$ derivative of the scaling function translation $i$. Once the derivative is obtained at integer values, the scale relation can be applied for any $x=k / 2^{j}$.

\section{Connection Coefficients}

The process of solving a DE requires the calculation of inner products of the basis functions and their derivatives $\left(n_{1}, n_{2}\right)$. These inner products are defined as connection coefficients and are given by:

$$
\Gamma_{i, j}^{n_{1}, n_{2}}=\int_{0}^{1} \varphi^{\left(n_{1}\right)}(\xi-i) \varphi^{\left(n_{2}\right)}(\xi-j) d \xi
$$

Dilation and translation properties allow the calculation of connection coefficients within the interval [ $\left[\begin{array}{ll}0 & 1\end{array}\right]$ to be summarized by the solution of an eigenvalue problem based only on filter coefficients (Zhou and Zhang, 1998):

$$
\begin{aligned}
& \left(\mathbf{P}-\frac{1}{2^{n_{1}+n_{2}-1}} \mathbf{I}\right) \boldsymbol{\Gamma}^{n_{1}, n_{2}}=0 \\
& P_{i, j: k, l}=a_{k-2 i} a_{l-2 j}+a_{k-2 i+1} a_{l-2 j+1}
\end{aligned}
$$

Since Eq. (8) leads to an infinite number of solutions, there is the need for a normalization rule that provides a unique eigenvector. This solution comes with the inclusion of an adapted version of the moment equation mentioned before:

$$
\begin{aligned}
& \sum_{i} \sum_{j} M_{i}^{k} M_{j}^{k} \Gamma_{i, j}^{n_{1}, n_{2}} \\
= & \frac{(k !)^{2}}{\left(k-n_{1}\right) !\left(k-n_{2}\right) !\left(2 k-n_{1}-n_{2}+1\right)}
\end{aligned}
$$

\section{Deslauriers-Dubuc Interpolating Wavelets}

The term interpolet was first used to designate wavelets with interpolating characteristics. The basic characteristics of interpolating wavelets require that the mother scaling function satisfies the following condition (Shi et al., 1999):

$$
\varphi(k)=\delta_{0, k}=\left\{\begin{array}{ll}
1, & k=0 \\
0, & k \neq 0
\end{array}, \quad k \in \mathbb{Z}\right.
$$

The filter coefficients $c_{k}$ for Deslauriers-Dubuc scaling function of order $N$ can be obtained by an autocorrelation of the same order Daubechies' filter coefficients $\left(a_{m}\right)$.

$$
\begin{aligned}
& \varphi(x)=\sum_{k=1-N}^{N-1} c_{k} \varphi(2 x-i) \\
& c_{k}=\sum_{m=0}^{N-1} a_{m} a_{m-k}
\end{aligned}
$$

Interpolets satisfy the same requirements as other wavelets, specially the two-scale relation, which is fundamental for their use as interpolating functions in numerical methods. Figure 2 shows the Interpolet IN4. Its symmetry and interpolating properties are evident. There is only one integer abscissa which evaluates to a non-zero value.

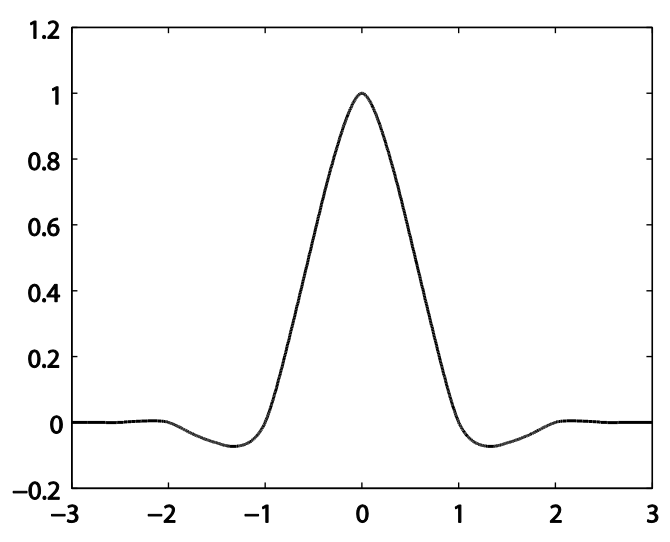

Figure 2. Interpolet IN4 scaling function.

All expressions used for the calculation of derivatives, connection coefficients and moments of 
Daubechies scaling functions can be applied to Interpolets. Of course, due to the correlation, the support $[0 ; N-1]$ in the expressions for Daubechies becomes $[1-N ; N-1]$ for Interpolets.

\section{WAVELET-GALERKIN METHOD}

The numerical solution of differential equations is one of the possible applications of the wavelet theory. The Wavelet-Galerkin Method (WGM) results from the use of wavelet scaling functions as the interpolating basis in a traditional Galerkin scheme. In the following sections, the WGM will be applied to solve one and two-dimensional Poisson's equations.

\section{Poisson's equation in 1-D (Truss equation)}

The one-dimensional Poisson's equation is equivalent to the differential equation of an axially loaded bar (truss element):

$$
\frac{d^{2} u}{d \xi^{2}}=f(\xi)
$$

Using interpolets as a function basis, multiplying by $\varphi(\xi-\mathrm{i})$ and integrating in [0 1] gives:

$$
\begin{aligned}
& u(\xi)=\sum_{j} d_{j} \varphi(\xi-j) \rightarrow \frac{d^{2} u}{d \xi^{2}}=\sum_{j} d_{j} \varphi^{\prime \prime}(\xi-j) \\
& \sum_{j} d_{j} \int_{0}^{1} \varphi(\xi-i) \varphi^{\prime \prime}(\xi-j) d \xi=\int_{0}^{1} f(\xi) \varphi(\xi-i) d \xi
\end{aligned}
$$

The system in Eq. (13) is in wavelet space and can be written in matrix form:

$$
\mathbf{k d}=\mathbf{f}, \quad \mathbf{k}=\Gamma^{02}
$$

Vector $\mathbf{f}$ can have an analytical solution depending on the function $f(\xi)$. The matrix $\mathbf{k}$ is composed by connection coefficients, which are obtained analytically for interpolets.

\section{Boundary conditions}

Some algebraic transformation needs to be performed in order to impose essential boundary conditions. Usually, Lagrange multipliers can be employed and they may or may not have physical meaning. After integration by parts and simplification:

$$
\begin{aligned}
& {\left[\begin{array}{lll}
\Gamma^{\mathbf{1 1}} & \boldsymbol{\Phi}(\mathbf{0})^{\mathrm{T}} & \boldsymbol{\Phi}(\mathbf{1})^{\mathrm{T}}
\end{array}\right]\left\{\begin{array}{c}
\mathbf{d} \\
u^{\prime}(0) \\
-u^{\prime}(1)
\end{array}\right\}=-\mathbf{f}} \\
& \Phi(0)_{j}=\varphi(-j) \\
& \Phi(1)_{j}=\varphi(1-j)
\end{aligned}
$$

The values for $u^{\prime}(0)$ and $u^{\prime}(1)$ can be both unknown or not. If one of them is known then it can be passed to the right side of the equation. Suppose one knows the value for $u^{\prime}(1)$, then the system becomes:

$$
\left[\begin{array}{ll}
\Gamma^{11} & \boldsymbol{\Phi}(\mathbf{0})^{\mathrm{T}}
\end{array}\right]\left\{\begin{array}{c}
\mathbf{d} \\
u^{\prime}(0)
\end{array}\right\}=-\mathbf{f}+\boldsymbol{\Phi}(\mathbf{1})^{\mathrm{T}} u^{\prime}(1)
$$

If this form is used, boundary conditions can be imposed in a similar form as in meshless methods (Nguyen et al., 2008), adding a transpose of each vector in order to obtain a square matrix. In this case, $\lambda$ is a vector of Lagrange multipliers. Another advantage of this form is that the basis only needs to have a first derivative. This allows the use of lower order wavelets. For most scaling functions (including Daubechies and Interpolets), the rank of the stiffness matrix is one unit less than its size, i. e., only one boundary condition needs to be imposed for the system to have a solution. Nevertheless, one needs to have at least two conditions for the system (for example, the displacement at one side and the derivative at the other). Denoting by $\mathbf{g}$ the matrix containing boundary conditions:

$$
\left[\begin{array}{cc}
\Gamma^{11} & \mathbf{g}^{\mathrm{T}} \\
\mathbf{g} & 0
\end{array}\right]\left\{\begin{array}{l}
\mathbf{d} \\
\boldsymbol{\lambda}
\end{array}\right\}=-\left\{\begin{array}{l}
\mathbf{f} \\
0
\end{array}\right\}
$$

In the case of a truss equation, the derivatives in Eq. (15) have physical meaning: they are the strains at $x=0$ and $x=1$, and therefore they are proportional to the stress at those points. In a truss, generally if a displacement is known, the applied force in its direction is unknown and vice-versa. For instance, in a truss with axial stiffness $E A$, with the left-end fixed $(u(0)=0)$ and an applied compressive force $F$ at the right-end $\left(\mathrm{F}=E A u^{\prime}(1)\right)$, the reaction force $R$ at $x=0$ and the displacement at $x=1$ are unknown. The system would become:

$$
\left[\begin{array}{ll}
\boldsymbol{\Gamma}^{\mathbf{1 1}} & \boldsymbol{\Phi}(\mathbf{0})^{\mathrm{T}} \\
\boldsymbol{\Phi}(\mathbf{0}) & 0
\end{array}\right]\left\{\begin{array}{c}
\mathbf{d} \\
\frac{R}{E A}
\end{array}\right\}=\left\{\begin{array}{c}
-\mathbf{f}+\boldsymbol{\Phi}(\mathbf{1})^{\mathrm{T}} \frac{F}{E A} \\
0
\end{array}\right\}
$$

In the more general case the system is obtained as in the following expression:

$$
\left[\begin{array}{lll}
\boldsymbol{\Gamma}^{\mathbf{1 1}} & \boldsymbol{\Phi}(\mathbf{0})^{\mathrm{T}} & \boldsymbol{\Phi}(\mathbf{1})^{\mathrm{T}} \\
\boldsymbol{\Phi}(\mathbf{0}) & 0 & 0 \\
\boldsymbol{\Phi}(\mathbf{1}) & 0 & 0
\end{array}\right]\left\{\begin{array}{c}
\mathbf{d} \\
u^{\prime}(0) \\
u^{\prime}(1)
\end{array}\right\}=\left\{\begin{array}{c}
-\mathbf{f} \\
u(0) \\
u(1)
\end{array}\right\}
$$

The last equations of the system in Eq. (19) are prescribed displacement equations. If interpolets are used, then the row and column of the correspondent degree of freedom can be removed, since the equations will become $d_{j}=u(0)$ and $d_{j+1}=u(1)$. It's important to emphasize that the system described 
above is not the weak form of the DE: it is equivalent to the conventional strong form system and was obtained through integration by parts and not by using an energy approach.

\section{Poisson's equation in 2-D}

For the solution of the two-dimensional Poisson's equation in wavelet space, a similar procedure is adopted. Some extra algebraic transformations have to be performed, leading to some interesting findings. The two-dimensional equation is given by:

$$
\frac{\partial^{2} u}{\partial \xi^{2}}+\frac{\partial^{2} u}{\partial \eta^{2}}=f(\xi, \eta)
$$

Provided that function $f$ is writable in separate variables and applying the same form of solution used in 1-D, the following can be written:

$$
\begin{aligned}
\left(\boldsymbol{\Gamma}^{\mathbf{0 2}} \otimes\right. & \left.\boldsymbol{\Gamma}^{\mathbf{0 0}}+\boldsymbol{\Gamma}^{\mathbf{0 0}} \otimes \boldsymbol{\Gamma}^{\mathbf{0 2}}\right) \boldsymbol{\alpha} \\
& =\int_{0}^{1} \boldsymbol{\Phi}^{\mathrm{T}} f(\xi) d \xi \otimes \int_{0}^{1} \boldsymbol{\Phi}^{\mathrm{T}} f(\eta) d \eta
\end{aligned}
$$

In Eq. (21), the symbol $\otimes$ indicates Kronecker product. Using the same integration by parts and rearranging the terms, the solution can be achieved by the following system:

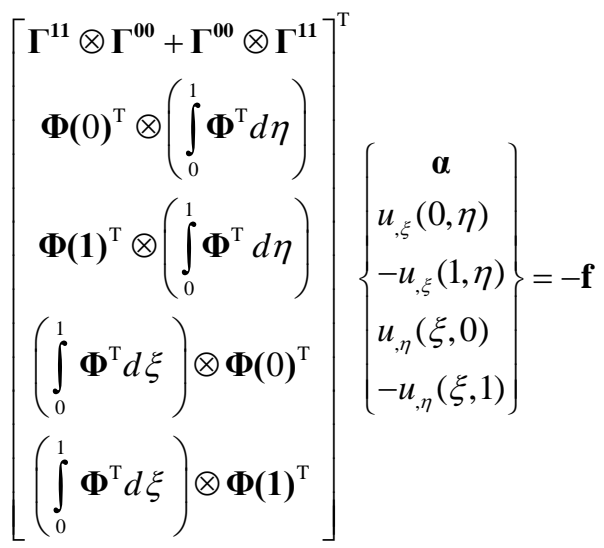

\section{EXAMPLES}

To validate the formulation, one and twodimensional examples were implemented and are shown in subsections below.

\section{1-D Poisson's equation with analytical solution}

As a first example, the one-dimensional form of Poisson's equation is solved. To test the capability of dealing with high order functions, a nonhomogeneous cubic function is used:

$$
\frac{d^{2} u}{d \xi^{2}}=1+x+x^{2}+x^{3}
$$

The particular solution for this equation is:

$$
u_{p}(x)=\frac{x^{2}}{2}+\frac{x^{3}}{6}+\frac{x^{4}}{12}+\frac{x^{5}}{20}
$$

To simulate the behavior of a truss element subjected to a force given by Eq. (23), boundary conditions were set as $u(0)=0$ and $u^{\prime}(1)=0$. The solution is given in Fig. 3. The convergence of the method was studied and compared to a Finite Differences (FDM) solution, as shown in Fig. 4. Interpolet IN4 was used in all examples.

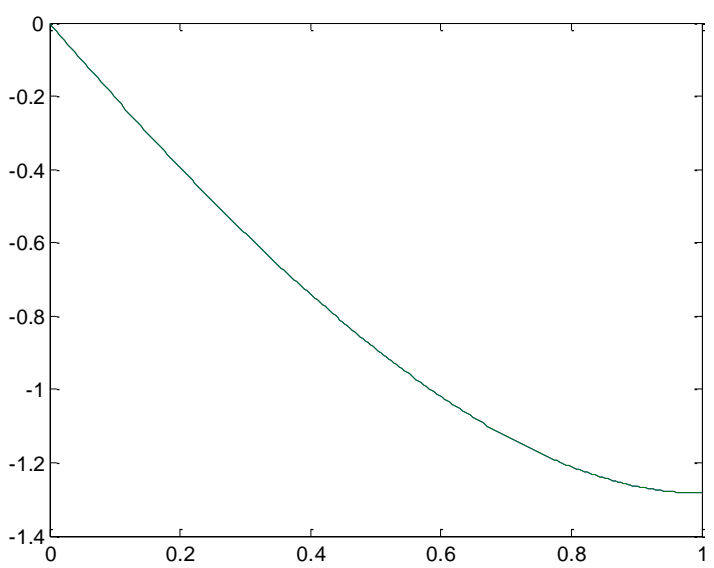

Figure 3. Solution for Example 1.

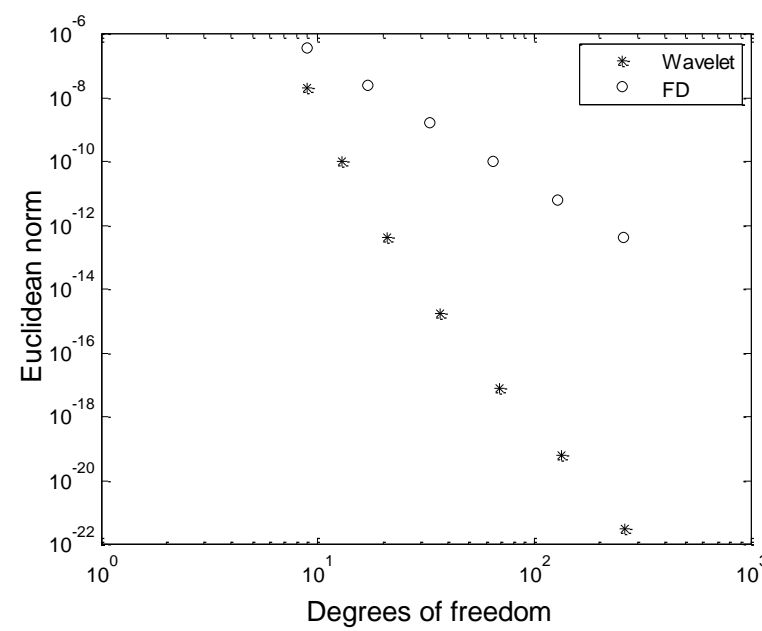

Figure 4. Convergence rate for Example 1.

\section{1-D Poisson's equation with random "noise"}

Normally, scattered data is provided by field measurements and a good way to subject this method to this kind of situation is to provide "noisy" data. The characteristics of this noise function are zero mean value and variances $(\sigma)$ that range from 0.001 to 0.1 . The Poisson's equation to be solved was 
obtained by using a constant unity non-homogeneous function:

$$
\frac{d^{2} u}{d \xi^{2}}=1
$$

Using a noise variance of $\sigma=0.001$, the constant function is represented by wavelet scaling functions as shown in Fig. 5. A spline representation was also used by means of comparison purpose. The solution of the differential equation is shown in Fig. 6. It is clear that there is no distinction between analytical and numerical solutions.

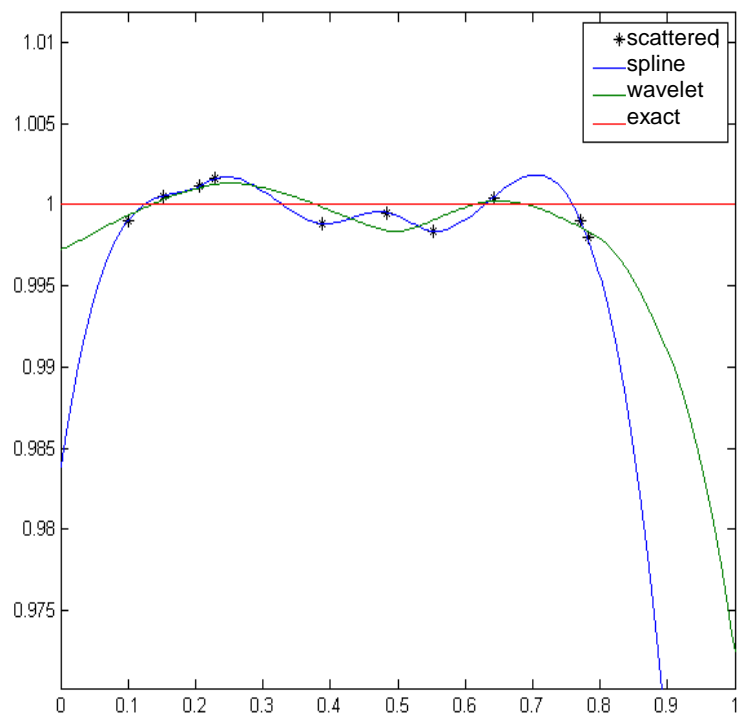

Figure 5. Representation of a constant function with added random noise $(\sigma=0.001)$.

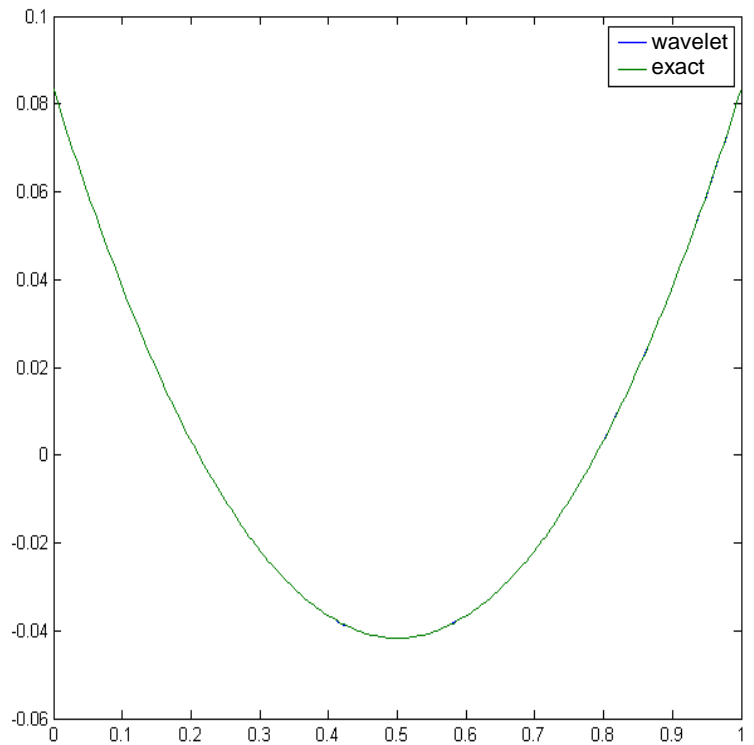

Figure 6. Wavelet and exact solutions with $\sigma=$ 0.001 .

Using a noise variance of $\sigma=0.1$, the constant function is represented by wavelet scaling functions as shown in Fig. 7. It is clear that wavelet representation is smoother than spline representation. The solution of the differential equation is shown in Fig. 8. Now there is a clear distinction between analytical and numerical solutions.

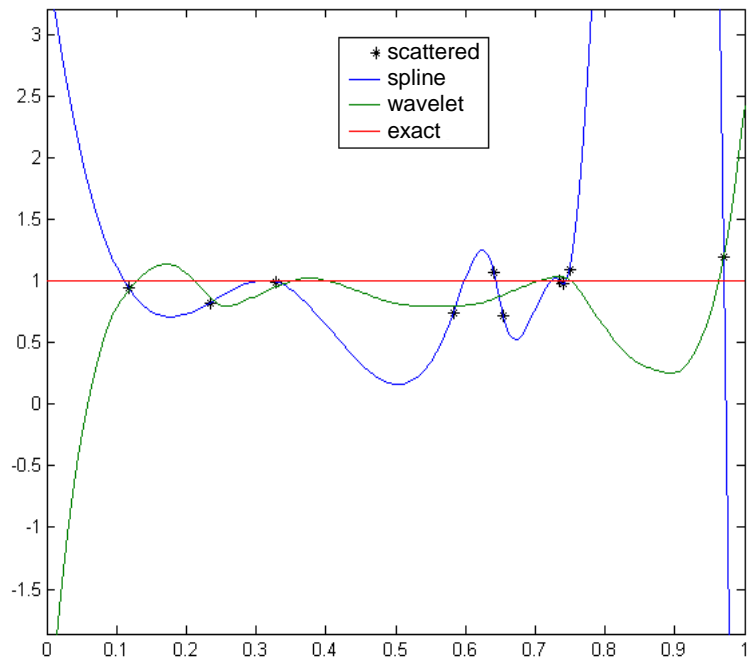

Figure 7. Representation of a constant function with added random noise $(\sigma=0.1)$.

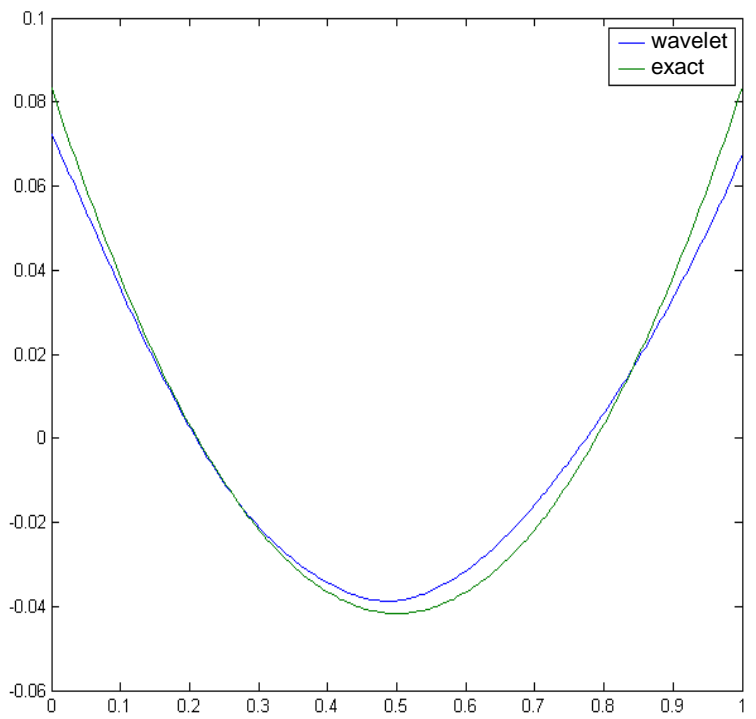

Figure 8. Wavelet and exact solutions with $\sigma=0.1$.

\section{2-D Poisson's equation with trigonometric functions}

Finally, the formulation was extended to a twodimensional problem, in which the non-homogeneous term is given by a trigonometric function:

$$
\frac{\partial^{2} u}{\partial x^{2}}+\frac{\partial^{2} u}{\partial y^{2}}=\cos \frac{x}{3} \sin \frac{x}{8} \cos \frac{y}{10} \sin y
$$

The domain within which the solution will be 
obtained is shown in Fig. 9. The non-homogeneous function is shown in Fig. 10. An arbitrary particular solution is given:

$$
\begin{aligned}
& u=\frac{3600}{12289} \sin \frac{5 x}{24} \sin \frac{9 y}{10}-\frac{3600}{20449} \sin \frac{11 x}{24} \sin \frac{11 y}{10} \\
& -\frac{3600}{14689} \sin \frac{11 x}{24} \sin \frac{9 y}{10}+\frac{3600}{18049} \sin \frac{5 x}{24} \sin \frac{11 y}{10}
\end{aligned}
$$

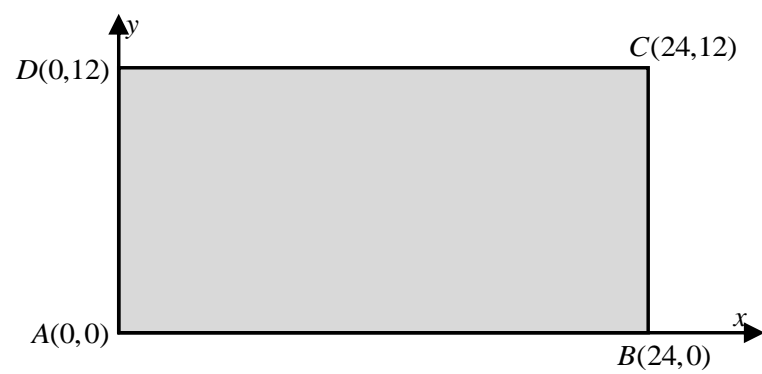

Figure 9. Rectangular domain for 2D example.

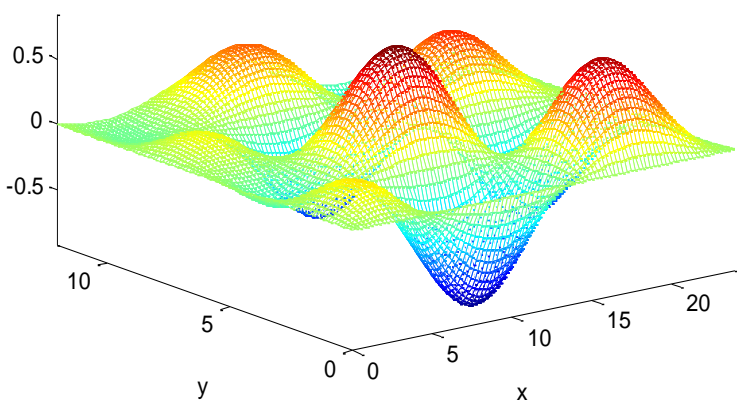

Figure 10. Non-homogeneous term in Eq. (26).

According to differential equations theory, any solution for Eq. (26) will contain Eq. (27). This particular solution is shown in Fig. 11.

Dirichlet boundary conditions were used in a way that the homogeneous solution becomes null. This is done by imposing boundary values of the particular solution to the total solution. Figure 12 shows a convergence study in which the error is measured using Euclidean Norm and compared to the errors given by the Finite Difference Method using second and fourth order formulations in spatial discretization.

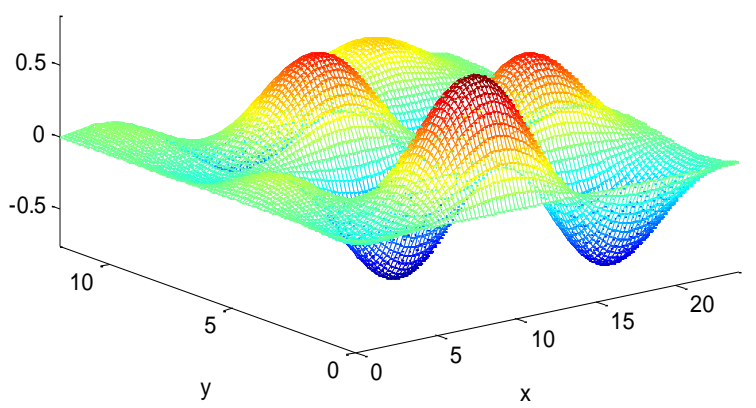

Figure 11. Particular solution given in Eq. (27).

\section{CONCLUSIONS}

This work presented the formulation and validation of the Wavelet-Galerkin Method using Deslauriers-Dubuc Interpolets for the solution of potential problems, namely the Poisson's equation.

As in the traditional FEM and other numerical methods, the accuracy of the solution can be improved either by increasing the level of resolution or the function order. Sometimes, lower order scaling functions at higher resolution can give better results than higher order functions at lower resolutions.

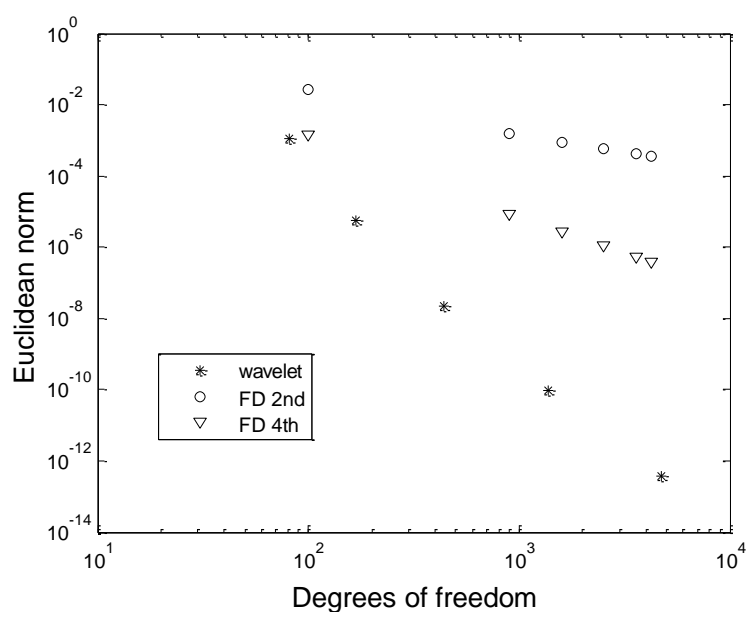

Figure 12. Convergence study for 2D example.

For two-dimensional problems, results were extremely good, although only regular geometry problems were studied. The extension of the method to irregular geometries remains a challenge.

When noisy data is provided, the method showed a very impressive ability to represent the solution acceptably. This is almost impossible to obtain with methods that depend on regularly distributed data, like the FDM. To use FDM, some interpolation would have to be performed beforehand.

Since the unknowns of the method are interpolation coefficients instead of nodal values, it is possible to obtain a smooth representation of solutions even with a reduced number of degrees of freedom.

All matrices involved can be stored and operated in a sparse form, since most of their components are null, thus saving computer resources. Due to the compact support of scaling functions, the sparseness of matrices increases along with the level of resolution.

\section{ACKNOWLEDGEMENTS}

Authors would like to thank CNPq and FAPERJ for their financial support.

\section{REFERENCES}


Burgos, R. B., Cetale Santos, M. A., and Silva, R. R., 2015, Analysis of Beams and Thin Plates Using the Wavelet-Galerkin Method, International Journal of Engineering and Technology, Vol. 7, pp. 261-266.

Chen, X., Yang, S., Ma, J., and He, Z., 2004, The Construction of Wavelet Finite Element and its Application, Finite Elements in Analysis and Design, Vol. 40, pp. 541-554.

Daubechies, I., 1988, Orthonormal Bases of Compactly Supported Wavelets, Communications in Pure and Applied Mathematics, Vol. 41, pp. 909-996.

Deslauriers, G., and Dubuc, S., 1989, Symmetric Iterative Interpolation Processes, Constructive Approximation, Vol. 5, pp. 49-68.

Latto, A., Resnikoff, L., and Tenenbaum, E., 1992, The Evaluation of Connection Coefficients of Compactly Supported Wavelets, in: Proceedings of the French-USA Workshop on Wavelets and Turbulence, New York, USA, pp. 76-89.

Lin, W., Kovvali, N., and Carin, L., 2005, Direct Algorithm for Computation of Derivatives of the Daubechies Basis Functions, Applied Mathematics and Computation, Vol. 170, pp. 10061013.

Ma, J., Xue, J., Yang, S., and He, Z., 2003, A Study of the Construction and Application of a Daubechies Wavelet-Based Beam Element, Finite Elements in Analysis and Design, Vol. 39, pp. 965975.

Nguyen, V. P., Rabczuk, T., Bordas, S., and Duflot, M., 2008, Meshless Methods: a Review and Computer Implementation Aspects, Mathematics and Computers in Simulation, Vol. 79, pp. 763-813.

Shi, Z., Kouri, D. J., Wei, G. W., and Hoffman, D. K., 1999, Generalized Symmetric Interpolating Wavelets, Computer Physics Communications, Vol. 119, pp. 194-218.

Zhou, X., and Zhang, W., 1998, The Evaluation of Connection Coefficients on an Interval, Communications in Nonlinear Science \& Numerical Simulation, Vol. 3, pp. 252-255. 